Review

ANA KATALENIĆ

Accepted 28. 11. 2021.

\title{
Asymptotes of Plane Curves - Revisited
}

\section{Asymptotes of Plane Curves - Revisited ABSTRACT}

In this paper we present a review of the basic ideas and results concerning asymptotic lines of plane curves. We discuss their different definitions, namely that of a limiting position of tangent lines, of the tangent line at infinity, and finally the one that requires that the distance between points of a curve and asymptotic line tends to 0 as the point moves along an infinite branch of the curve. We also recall the method of determining asymptotes of algebraic curves from the leading coefficients in their equation and provide examples.

Key words: plane curve, asymptote, limiting tangent line, tangent at infinity

MSC2010: 51-02

\section{Introduction}

Many plane curves have asymptotes. They are an inevitable part of the curve sketching. In this paper, the term asymptote will primarily refer to the asymptotic straight line, where, of course, there exist other asymptotic curves such as asymptotic parabolas or cubic curves, or asymptotic points.

In the first encounter with the notion an asymptote is very often described as a straight line that approaches a curve but never touches it which is a suitable description for the prototype school examples of curves, such as a hyperbola as the graph of a rational function $f(x)=1 / x$, the graph of an exponential and logarithmic function, or a hyperbola as a curve in analytic geometry. However, rigorous mathematical definition which developed through history includes the possibility that the curve intersects its asymptote or that it oscillates around the asymptote. In this historical development, we can mention Apollonius of Perga (262

\begin{abstract}
Asimptote ravninske krivulje - ponovni pogled SAŽETAK

$\mathrm{U}$ ovom radu dajemo pregled osnovnih ideja i rezultata vezanih uz asimptote ravninskih krivulja. Raspravljamo o njihovim različitim definicijama, naime, o definiciji kao o graničnom položaju tangenata, o definiciji kao o tangenti u beskonačnosti, te konačno o definiciji koja zahtijeva da udaljenost između točke krivulje i asimptote teži 0 kako se točka kreće duž beskonačne grane krivulje. Također se prisjećamo metode određivanja asimptota algebarskih krivulja iz vodećih koeficijenata u njihovoj jednadžbi te navodimo primjere.
\end{abstract}

Ključne riječi: ravninska krivulja, asimptota, granična tangenta, tangenta u beskonačnosti

BC-190 BC) who introduced the aforementioned description of an asymptote of a curve as "lines [a curve and its asymptote] which do not meet, in whatever direction they are produced" [21]. Much later, in his work on perspective, Desargues (1591-1661) took a different approach, namely that of projective geometry, and introduced asymptotes as tangent lines at infinity, whereas Newton (1643-1727) used asymptotes as the main tool in classification of cubics on the account of their points at infinity [1]. In historical calculus sources from the transition between $19^{\text {th }}$ and the $20^{\text {th }}$ century, an asymptote of a curve was given as the limiting position of a tangent line to the curve when the point of tangency moves away from the origin, or a line, the distance of which from a point in a curve diminishes as the point moves away from the origin [3, 4, 5, 6, 18, 22].

In school and early university mathematics asymptotes are considered for graphs of real (smooth) functions. There is no unique or "the best" choice of definition, which may be confirmed by the following quotations: 
"My own preference is for the limiting tangent definition, partly because I feel that asymptotes have something to do with tangents, and partly because it is easier to use than the rival definitions" ([8], p. 281);

and

"The limit of tangents may not exist, even when asymptotes exist [in the sense of definition by distance]. This fact shows that the limit of tangents is not a suitable definition of an asymptote" ([6], p. 91).

In our previous work we were interested in students' recollection of the notion of asymptote [13, 12, 11]. Motivated by the richness of the notion, our aim here is to present a mathematical review of the basic ideas and results concerning asymptotes of plane curves that goes beyond school requirements.

\section{Definitions of an asymptote}

Definition 1 [23] A line l is an asymptote to a curve if the distance from a point $P$ to the line $l$ tends to zero as $P$ tends to infinity along some unbounded part of the curve.

Definition 2 [9] Asymptotes are the limits of tangent lines when the point of contact tends to infinity.

Definition 3 [19] An asymptote of a plane curve is a tangent to the projective curve determined by it at a point at infinity, which tangent is not the line at infinity.

These definitions are not equivalent in general. In the following we first analyze what they imply.

Distance between points in Definition 1 is taken as the Euclidean distance. But if this distance tends to zero, then the vertical (for a horizontal or oblique asymptote) or the horizontal distance (for a vertical asymptote), also tends to zero [2, 6, 15]. The vertical or the horizontal distance is the absolute value of difference of the corresponding coordinates of a point on a curve and on an asymptotic line.

This consideration implies that, following Definition 1, a line $y=k x+l$ is an asymptote of a function $f: I \rightarrow \mathbb{R}$ of class $C^{1}$, where $I \subset \mathbb{R}$ is an open interval, if and only if $\lim _{x \rightarrow \infty}(f(x)-k x-l)=0[15]$. Now it follows

$k=\lim _{x \rightarrow \infty} \frac{f(x)}{x}, l=\lim _{x \rightarrow \infty}(f(x)-k x)$.

To use Definition 2, we start from a tangent line of a curve which is the graph of a function $f$ at a point $\left(x_{0}, f\left(x_{0}\right)\right)$, and given by

$$
y=f^{\prime}\left(x_{0}\right) x+f\left(x_{0}\right)-f^{\prime}\left(x_{0}\right) x_{0} .
$$

The graph of a function $f$ has a limiting tangent line if and only if the direction of the tangent line and its intercept with $y$ axis have limiting value, that is, the limits

$$
\lim _{x_{0} \rightarrow \infty} f^{\prime}\left(x_{0}\right), \lim _{x_{0} \rightarrow \infty}\left(f\left(x_{0}\right)-f^{\prime}\left(x_{0}\right) x_{0}\right)
$$

exist [4, 8]. If $\lim _{x_{0} \rightarrow \infty} f^{\prime}\left(x_{0}\right)=\infty$ the function might still have vertical asymptote.

We reason similarly for parametrized curves or curves given by implicit equations; to find a tangent line of a curve $c(t)=(u(t), v(t))$ or a curve given by $F(x, y)=0$ requires looking at the limiting value of the gradient $v^{\prime} / u^{\prime}$ or $-\frac{\partial F}{\partial x} / \frac{\partial F}{\partial y}$, and if the limit exists, then looking at the limiting value of the intercept of the tangent line on the axis [4, 8].

Definition 3 is set up in projective plane which is also a natural way of thinking about asymptotes. We extend $\mathbb{R}^{2}$ and assume the following correspondence between $\mathbb{R}^{2}$ and $\mathbb{P}^{2}$, which maps a point at infinity of the curve in the direction of $x \rightarrow \infty$ to the origin of the real plane

$(x, y) \mapsto[x, y, z] \mapsto[z, y, x]=\left[\frac{z}{x}, \frac{y}{x}, 1\right] \mapsto\left(\frac{z}{x}, \frac{y}{x}\right)$.

First arrow represents the mapping $P: \mathbb{R}^{2} \rightarrow \mathbb{P}^{2}, P(x, y)=$ $[x, y, z]$, where points in $\mathbb{P}^{2}$ are equivalence classes given with $[x, y, z]=\{(\alpha x, \alpha y, \alpha z), \alpha \in \mathbb{R}, \alpha \neq 0\}$. Second is projective transformation $T: \mathbb{P}^{2} \rightarrow \mathbb{P}^{2}, T[x, y, z]=[z, x, y]$, such that $T^{2}=i d$. Last is the mapping $R: \mathbb{P}^{2} \rightarrow \mathbb{R}^{2}$, $R[z, y, x]=(z, y)$. An asymptote, as the tangent at infinity, corresponds to the tangent line at the origin following the mapping in (2) [8, 10, 20].

Example 1. The curve $c$ given by the equation $F(x, y)=$ $y^{3}-x^{3}+1=0$ has the line $y=x$ as an asymptote in the sense of all definitions.

Def 1. Let $P\left(x_{P}, y_{P}\right)$ be a point on the curve $c$, and $d=$ $\frac{\left|x_{P}-y_{P}\right|}{\sqrt{2}}$ is the distance between the point $P$ on the curve $c$ and the line $y=x$. Since

$$
d=\frac{\left(x_{P}-\sqrt[3]{x_{P}^{3}-1}\right)}{\sqrt{2}} \rightarrow 0, \text { for } x_{P} \rightarrow \infty
$$

the line $y=x$ is an asymptote.

Def 2. The equation of the tangent line to the curve $c$ at point $P$ is

$$
\left(\frac{\partial F}{\partial x}\right)_{P}\left(x-x_{P}\right)+\left(\frac{\partial F}{\partial y}\right)_{P}\left(y-y_{P}\right)=0 .
$$

The direction of the tangent line in a point $\left(x_{P}, y_{P}\right)$ is given by $k_{P}=-\frac{\left(\frac{\partial F}{\partial x}\right)_{P}}{\left(\frac{\partial F}{\partial y}\right)_{P}}=\frac{3 x_{P}^{2}}{3 y_{P}^{2}}$, and the intercept of 
the tangent line with $y$ axis by $l_{P}=y_{P}+\frac{\left(\frac{\partial F}{\partial x}\right)_{P}}{\left(\frac{\partial F}{\partial y}\right)_{P}} \cdot x_{P}=$ $\frac{-3}{3 \cdot \sqrt[3]{x_{P}^{3}-1}}$, both having the limiting values for $x_{P} \rightarrow \infty$ as $k_{P} \rightarrow 1$ and $l_{P} \rightarrow 0$ respectively. The line $y=x$ is a limiting tangent line.

Def 3. The homogeneous equation of the curve $c$ in the projective plane $\mathbb{P}^{2}$ is given with $f(x, y, z)=y^{3}-x^{3}+$ $z^{3}=0$. The equation of the tangent line to the projective curve at point with homogeneous coordinates $P\left[x_{P}, y_{P}, z_{P}\right]$ is

$$
\left(\frac{\partial f}{\partial x}\right)_{P} x+\left(\frac{\partial f}{\partial y}\right)_{P} y+\left(\frac{\partial f}{\partial z}\right)_{P} z=0,
$$

that is, $\left(-3 x_{P}^{2}\right) x+\left(3 y_{P}^{2}\right) y+\left(3 z_{P}^{2}\right) z=0$. The point at infinity of the curve $f(x, y, z)=0$ has homogeneous coordinates $[1,1,0]$ and the equation of the tangent line to the curve at the point at infinity is $-3 x+3 y=0$, that is, the line $y=x$ in the real plane. This line is a tangent at infinity.

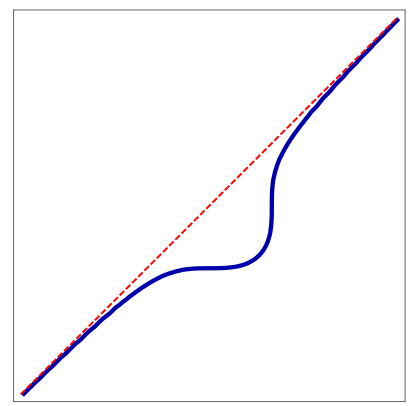

Figure 1: Example 1

Example 2. The graph of the function $f(x)=\frac{\sin x}{x}$ has the line $y=0$ as an asymptote in the sense of Definitions 1 and 3. but not in the sense of Definition 2

Def1. Since $k=\lim _{x \rightarrow \infty} \frac{f(x)}{x}=0$ and $l=\lim _{x \rightarrow \infty}(f(x)-k x)=$ $\lim _{x \rightarrow \infty} \frac{\sin x}{x}=0$, the line $y=0$ is the asymptote.

Def.2. The direction of the tangent line $f^{\prime}\left(x_{0}\right)=\frac{\cos x_{0}}{x_{0}}-$ $\frac{\sin x_{0}}{x_{0}^{2}} \rightarrow 0$ has a limiting value, but the intercept of the tangent line with the $y$ axis, $f\left(x_{0}\right)-f^{\prime}\left(x_{0}\right) x_{0}=$ $-\cos x_{0}$ has no limiting value as $x_{0} \rightarrow \infty$. The function has no limiting tangent line.

Def 3 . Following the correspondence (2) we obtain

$$
\begin{gathered}
(x, f(x)) \mapsto[x, f(x), 1] \mapsto[1, f(x), x]=\left[\frac{1}{x}, \frac{f(x)}{x}, 1\right] \\
\mapsto\left(t, t \cdot f\left(\frac{1}{t}\right)\right), t=\frac{1}{x} .
\end{gathered}
$$

The tangent of the function $F(t)=t \cdot f\left(\frac{1}{t}\right)$, for $t=0$ and $F(0)=\lim _{x \rightarrow \infty} \frac{f(x)}{x}=0$, is the limit of the secants through point $(0,0)$ and $(F(t), t)$, as $t \rightarrow 0$. Since $\lim _{t \rightarrow 0} \frac{F(t)}{t}=\lim _{t \rightarrow 0} f\left(\frac{1}{t}\right)=\lim _{x \rightarrow \infty} f(x)=0$, the limit of the secants is $y=0$ which corresponds back to the line $y=0$ as tangent at infinity.

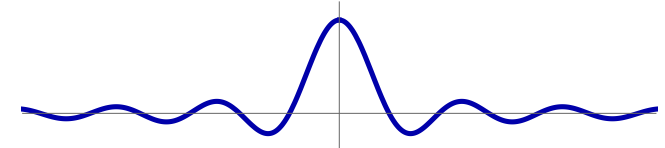

Figure 2: Example 2

To analyze relations between Definitions 1, 2 and 3, we introduce $c=(u(t), v(t))$ as a (parametrized) curve in $\mathbb{R}^{2}$, with continuous first derivatives and infinite branch in the direction $t \rightarrow t_{0}$, and without loss of generality $u(t) \rightarrow \infty$.

Theorem 1 (1) If a line lis an asymptote of the curve $c$ in the sense of Definition 2, then $l$ is an asymptote of the curve $c$ in the sense of Definition 1 .

(2) If a line $l$ is an asymptote of the curve $c$ in the sense of Definition 2 then $l$ is an asymptote of the curve c in the sense of Definition 3

(3) A line $l$ is an asymptote of the curve $c$ in the sense of Definition 3 if and only if $l$ is an asymptote of the curve $c$ in the sense of Definition 1 .

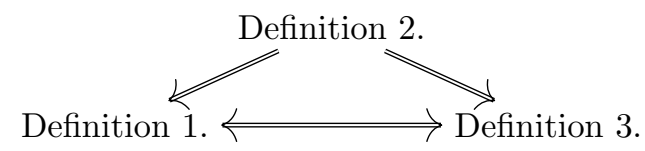

Figure 3: Relationship between the three definitions of an asymptote

We provide the proof of the Theorem 1

(1) Proof. Assume $c$ has a limiting tangent line, that is,

$y=\lim _{t \rightarrow t_{0}} \frac{v^{\prime}(t)}{u^{\prime}(t)} \cdot x+\lim _{t \rightarrow t_{0}}\left(v(t)-\frac{v^{\prime}(t)}{u^{\prime}(t)} \cdot u(t)\right)$

where $k=\lim _{t \rightarrow t_{0}} \frac{v^{\prime}(t)}{u^{\prime}(t)}$ and $l=\lim _{t \rightarrow t_{0}}\left(v(t)-\frac{v^{\prime}(t)}{u^{\prime}(t)} \cdot u(t)\right)$.

The distance between the limiting tangent line and point on curve $c$ is given by

$d=\frac{|k \cdot u(t)-v(t)+l|}{\sqrt{k^{2}+1}}$. 
Since

$$
\begin{aligned}
\lim _{t \rightarrow t_{0}}(v(t)-k \cdot u(t)) & =\lim _{t \rightarrow t_{0}} \frac{\frac{v(t)}{u(t)}-k}{\frac{1}{u(t)}} \\
& =\lim _{t \rightarrow t_{0}} \frac{\frac{v^{\prime}(t) \cdot u(t)-v(t) \cdot u^{\prime}(t)}{(u(t))^{2}}}{-\frac{u^{\prime}(t)}{(u(t))^{2}}} \\
& =\lim _{t \rightarrow t_{0}}\left(v(t)-\frac{v^{\prime}(t)}{u^{\prime}(t)} \cdot u(t)\right)=l,
\end{aligned}
$$

then $d \rightarrow 0$ as $t \rightarrow t_{0}$, and the limiting tangent given in (3) is an asymptote in the sense of Definition 1 . $\square$

(2) Proof. Assume $c$ has a limiting tangent line $y=$ $k x+l$ given in $(3)$. By embedding $\mathbb{R}^{2}$ in $\mathbb{P}^{2}$ the homogenenous coordinates of a point on the curve $c$ are

$$
(u(t), v(t)) \mapsto[u(t), v(t), 1]=\left[1, \frac{v(t)}{u(t)}, \frac{1}{u(t)}\right]
$$

and the point at infinity of the curve $c$ is

$$
\left[1, \lim _{t \rightarrow t_{0}} \frac{v(t)}{u(t)}, \lim _{t \rightarrow t_{0}} \frac{1}{u(t)}\right]=(1, k, 0)
$$

since by l'Hospital rule $k=\lim _{t \rightarrow t_{0}} \frac{v^{\prime}(t)}{u^{\prime}(t)}=\lim _{t \rightarrow t_{0}} \frac{v(t)}{u(t)}$.

Tangent at infinity corresponds to the limit of the secants (or the chords [6, 8]) joining the point at infinity of the curve with an arbitrary point on the projective curve, as it tends to the point at infinity. Following (2)

$$
\begin{aligned}
(u(t), v(t)) & \mapsto[u(t), v(t), 1] \mapsto \\
& \mapsto[1, v(t), u(t)]=\left[\frac{1}{u(t)}, \frac{v(t)}{u(t)}, 1\right]
\end{aligned}
$$

and the chord joining points $(0, k)$ and $\left(\frac{1}{u(t)}, \frac{v(t)}{u(t)}\right)$ on the corresponding curve is

$$
\begin{aligned}
y-k & =\frac{\frac{v(t)}{u(t)}-k}{\frac{1}{u(t)}-0}(z-0) \\
y & =(v(t)-k \cdot u(t)) \cdot z+k
\end{aligned}
$$

which transforms back into $y=k \cdot x+(v(t)-k \cdot u(t))$. The tangent at infinity is thus given by

$y=k \cdot x+\lim _{t \rightarrow t_{0}}(v(t)-k \cdot u(t))$.

Since $\lim _{t \rightarrow t_{0}}(v(t)-k \cdot u(t))=l$ the tangent at infinity given in (4) coincides with the limiting tangent given in (3).
(3) Proof. Let $y=k x+l$ be a tangent at infinity of a curve $c$ given in (4). Then $k=\lim _{t \rightarrow t_{0}} \frac{v(t)}{u(t)}$, and $l=\lim _{t \rightarrow t_{0}}(v(t)-k \cdot u(t))$. Distance between the tangent at infinity and a curve $c$ is given by

$d=\frac{|k \cdot u(t)-v(t)+l|}{\sqrt{k^{2}+1}} \rightarrow 0$ as $t \rightarrow t_{0}$.

Therefore, the tangent at infinity given in (4) is an asymptote in the sense of Definition 1

To show the converse, let $y=k x+l$ be an asymptote of a curve $c$ in the sense of Definition 1 , that is,

$$
d=\frac{|k \cdot u(t)-v(t)+l|}{\sqrt{k^{2}+1}} \rightarrow 0 \text { as } t \rightarrow t_{0} .
$$

Then

$$
\lim _{t \rightarrow t_{0}}(v(t)-k \cdot u(t))=l \text { and } \lim _{t \rightarrow t_{0}} \frac{v(t)}{u(t)}=k
$$

which correspond to the coefficients of a tangent at infinity given in (4).

However, as stated in [8] for algebraic curves the following theorem holds.

Theorem 2 Definitions 1, 2, 3 are equivalent in the case of algebraic curves.

\section{Methods of finding asymptotes of alge- braic curves}

Definitions we discussed provide different ways how to determine asymptotes of plane curves. We summarize that the most common way how to determine slant asymptotes of a function graph is to look for them as $y=k x+l$ where coefficients $k, l$ are given by (1). Furthermore, in the special case of a rational function $f(x)=\frac{P(x)}{Q(x)}$ its (linear or curvilinear) asymptote is the quotient of the polynomials in the numerator and denominator. For example, a function $f(x)=\frac{x^{2}+1}{x-1}$ can be rewritten as $f(x)=x+1+\frac{2}{x-1}$ which enables to recognize the equation of its slant asymptote as $y=x+1$. Its vertical asymptote appears as the zero of the denominator $x=1$ (see Figure 4 on the right). This procedure also gives asymptotes of algebraic curves when their equation can be expressed in the suitable form by expressing, for instance, $y$ by $x$.

Generally, finding asymptotes of a real algebraic plane curve reduces to finding corresponding tangent lines at the points at infinity of the projective curve (see Example 1 and [10]) or at the origin for the corresponding curves obtained by projective transformations (see Examples 2, 4 and 5, and [17, 20]). In the purely algebraic context, finding 
asymptotes comes down to determining lines that reduce the degree of the equation of algebraic curve. Regardless of the chosen definition, an asymptote of the curve is line that intersects the curve in at least two coincident points at an infinite distance [2, 4, 5, 6, 7].

In the case of a hyperbola $b^{2} x^{2}-a^{2} y^{2}=a^{2} b^{2}$, if we analyze a number of common (real) points with a line $y=k x+l$ (which can be 0,1 or 2 ), we arrive at the quadratic equation $\left(b^{2}-a^{2} k^{2}\right) x^{2}-2 a^{2} k l x-a^{2} l^{2}-a^{2} b^{2}=0$ with one solution in $x$ if and only if $k^{2} a^{2}-b^{2}=l^{2}$. This is known as the tangency condition. However, when the leading coefficient vanishes, that is $b^{2}-a^{2} k^{2}=0$ (implying that $k= \pm \frac{b}{a} \neq 0$ ), and when the next coefficient vanishes as well, giving $l=0$, we arrive at the known equation of an asymptote of the hyperbola as $y= \pm \frac{b}{a} x$. This method of determining the asymptote can also be generalized. In [14] conditions for a quadratic curve

$a_{1} x^{2}+a_{2} x y+a_{3} y^{2}+b_{1} x+b_{2} y+c=0$

to have asymptotes are explored, which can be further interpreted as conditions that ensure that a quadratic curve is a hyperbola. By substituting $y=k x+l$ in (5) we arrive at the quadratic equation in $x$, and by equating the coefficients of $x^{2}$ and $x$ to 0 , while the constant coefficient is not 0 , we obtain the asymptotes of a curve. Summarizing [14], if

A1 $a_{2}^{2}-4 a_{1} a_{3}>0$ and the equation (5) cannot be factorised into linear factors, it represents a hyperbola with asymptotes $y=k x+l$, where $k$ and $l$ are solutions of equations $a_{1}+a_{2} k+a_{3} k^{2}=0$ and $a_{2} l+$ $2 a_{3} k l+b_{1}+b_{2} k=0$.

A2 $a_{3}=0, a_{2} \neq 0$ and the equation (5) cannot be factorised into linear factors, it represents a hyperbola with a vertical asymptote $x=-\frac{a_{1}}{a_{2}}$ and a slant asymptote $y=-\frac{a_{1}}{a_{2}} x+\frac{a_{1} b_{2}-a_{2} b_{1}}{a_{2}^{2}}$.

\section{Example 3.}

(i) Let the curve be given by $-2 x^{2}+x y+y^{2}-y-1=0$ (see Figure 4 above)

- From A1 it follows that the curve is a hyperbola and the coefficients of its asymptotes satisfy $-2+k+k^{2}=0$ and $l+2 k l-k=0$. It follows $k_{1}=1, k_{2}=-2$ and $l_{1}=\frac{1}{3}, l_{2}=\frac{2}{3}$. Asymptotes are $y=x+\frac{1}{3}$ and $y=-2 x+\frac{2}{3}$.

- The equation of the curve can be written as $(y-x)(y+2 x)=y+1$. We have

$$
y-x=\frac{y+1}{y+2 x} \underset{x \rightarrow \infty, y \rightarrow x}{\longrightarrow} \frac{x+1}{3 x}=\frac{1}{3}+\frac{1}{x} .
$$

The asymptote is $y-x=\frac{1}{3}$, and similarly the other asymptote is $y+2 x=\frac{2}{3}$.

(ii) Let the curve be given by $-x^{2}+x y-y-1=0$ (see Figure 4 below)

- From A2 it follows that the curve is a hyperbola with the vertical asymptote $x=1$ and a slant asymptote $y=x+1$.

- The equation of the curve can be written as $x(y-x)=y+1$, and

$$
y-x=\frac{y+1}{x} \underset{x \rightarrow \infty, y \rightarrow x}{\longrightarrow} \frac{x+1}{x}=1+\frac{1}{x} .
$$

The asymptote is $y=x+1$. From $-\frac{x^{2}}{y}+x-$ $1-\frac{1}{y}=0$, it follows that when $y \rightarrow \infty$, then $x-1 \rightarrow 0 . x=1$ is vertical asymptote.
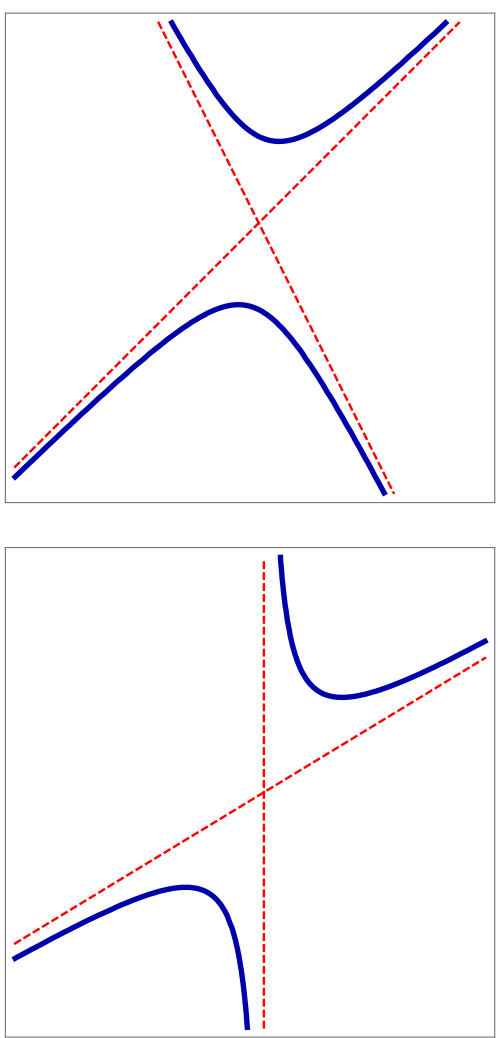

Figure 4: Example 3

We describe methods of finding asymptotes of general algebraic curves. Let $c$ be an algebraic curve given by an equation.

$F(x, y)=P_{n}(x, y)+P_{n-1}(x, y)+\cdots+P_{1}(x, y)+P_{0}=0$

where $P_{m}(x, y)$ is a term of degree $m, P_{n}=\sum_{i=0}^{m} a_{m, i} x^{m-i} y^{i}$. 
Assume $x \rightarrow \infty$. Substituting $y=k x+l$ results with an equation of degree $n$ in $x$. For the line $y=k x+l$ to be an asymptote of the curve (6) the two coefficients of the top degree in the resulting equation must vanish, thus providing the coefficient $k$ as the root of the leading term $P_{n}(x, y)$ in $(x, y)=(1, k)$. This method for finding asymptotes is reported in different sources, and in [6] it is summarized with the following theorem:

Theorem 3 The line $y=k x+l$ is an asymptote of the algebraic curve (6) if and only if

(1) $k$ is a real root of equation $P_{n}(1, t)=0$,

(2) for chosen $k$, the coefficient $l$ is a root of equation $\psi(s, k)=0$, where

$\phi(s, k, x)=\psi(s, k)+\frac{1}{x} \psi_{1}(s, k)+\frac{1}{x^{2}} \psi_{2}(s, k)+\cdots=0$

and $\phi$ is obtained by reduction from $F(x, s+k x)=0$, and

(3) for chosen $k$ and $l$, equation $\phi(l+\varepsilon, k, x)=0$ admits real root $\varepsilon$ such that $\varepsilon \rightarrow 0$ for $x \rightarrow \infty$.

The equation of the curve (6) can be expressed as

$$
\begin{aligned}
& x^{n} P_{n}\left(1, \frac{y}{x}\right)+x^{n-1} P_{n-1}\left(1, \frac{y}{x}\right)+x^{n-2} P_{n-2}\left(1, \frac{y}{x}\right)+\cdots \\
& \quad+x P_{1}\left(1, \frac{y}{x}\right)+P_{0}=0 .
\end{aligned}
$$

By substituting $\frac{y}{x}=k+\frac{l}{x}$ and by Taylor's theorem we obtain [5, 6, 15]

$$
\begin{aligned}
& x^{n} P_{n}(1, k)+x^{n-1}\left(P_{n-1}(1, k)+l \cdot P_{n}^{\prime}(1, k)\right)+ \\
& \quad x^{n-2}\left(P_{n-2}(1, k)+l \cdot P_{n-1}^{\prime}(1, k)+\frac{l^{2}}{2} P_{n}^{\prime \prime}(1, k)\right)+\cdots=0 .
\end{aligned}
$$

The simplest situation is when $k$ is a simple root of $P_{n}(1, t)=0$. Then $P_{n}^{\prime}(1, k) \neq 0$ and for $x \rightarrow \infty$ the form $\psi(s, k)$ in condition (3) of Theorem 3 reduces to

$$
P_{n-1}(1, k)+s \cdot P_{n}^{\prime}(1, k) .
$$

But if $k$ is $r$-tuple root of $P_{n}(1, t)=0$, depending on the values of $P_{i}(1, k)$ and $P_{i}^{(j)}(1, k)$, and corresponding form $\psi(s, k)$, different situations can occur. For example, different branches can correspond to the same asymptote, the curve can have parallel asymptotes, or the curve can have no asymptotes. In the latter case, the curve could have a parabolic branch with a parabolic asymptote, or a general curvilinear asymptote, when the condition 3 of Theorem 3 fails.

The condition (3) of Theorem 3 is the necessary condition for the line $y=k x+l$ to be the asymptote of the curve $(6)$, that is, that the curve must have an infinite branch in the direction of the line $y=k x+l$. Note that the method of leading coefficients following from conditions (3) and (3) in the Theorem 3 would still provide a line as an asymptote even if the curve has no infinite branch (see Example 4). Nunnemacher [16] noted that such spurious asymptotes correspond to the complex branch of the curve. He provided a simpler method for exploring asymptotes of algebraic curves, focused on the multiplicity of the factor $a x+b y$ (rather than $y-k x$ ) in the term of the top degree in (6). This method simplifies the calculation, and parallel asymptotes and parabolic branches are easily discerned (see Examples 4 and 5) but the theorem does not resolve the issue of spurious asymptotes when no real branch can be associated with the obtained line.

Theorem 4 Suppose that ax + by is a factor of the top degree form $P_{n}$ of multiplicity $m$ with $a$ and $b$ real. Let $r \leq m$ denote the largest integer with the property that there exist polynomials $Q_{j}(x, y)$ for $n-r+1 \leq j \leq n$ satisfying the conditions:

$$
\begin{array}{r}
P_{n}(x, y)=(a x+b y)^{r} Q_{n}(x, y), \\
P_{n-1}(x, y)=(a x+b y)^{r-1} Q_{n-1}(x, y), \ldots,
\end{array}
$$

and finally $P_{n-r+1}(x, y)=(a x+b y) Q_{n-r+1}(x, y)$.

Then associated with the factor ax + by is a set of at most $r$ possible asymptotes $a x+b y=t_{0}$, where $t_{0}$ is a real root of the equation

$$
\begin{aligned}
& t^{r} Q_{n}(b,-a)+t^{r-1} Q_{n-1}(b,-a)+\cdots+t Q_{n-r+1}(b,-a) \\
& \quad+P_{n-r}(b,-a)=0 .
\end{aligned}
$$

All real asymptotes to the curve $c$ arise in this way as $a x+$ by ranges over the real linear factors of $P_{n}(x, y)$. If $r>1$ it may happen that some of these lines are spurious asymptotes.

We illustrate the methods and issues with asymptotes of algebraic curves in the following examples.

Example 4 Let $c$ be the curve given by the equation

$$
F(x, y)=x^{4}-2 x^{2} y^{2}+y^{4}+x^{3}-2 x^{2} y+x y^{2}+1=0 .
$$

Following Theorem 3 , we find $k_{1}=1, k_{2}=-1$ as the zeros of the leading term $P_{4}(1, t)=(t-1)^{2}(t+1)^{2}$ in the equation of the curve. We substitute for $y=k x+l$ :

- If $y=x+l$, then $F(x, x+l)=l^{2}(2 x+l)^{2}+x \cdot l^{2}+1=$ 0 .

The condition (3) of Theorem 3 implies $4 l^{2}+$ $\frac{1}{x}\left(4 l^{3}+l^{2}\right)+\frac{1}{x^{2}}\left(l^{4}+1\right)=0$ and the coefficient $l$ of the line derives from $\psi=4 l^{2}=0$, therefore $l=0$.

This is spurious asymptote since $c$ has no a real branch for $x \rightarrow \infty$ in the direction of the line $y-x=$ 0 . 
- If $y=-x+l$, then $F(x,-x+l)=(-2 x+l)^{2} l^{2}+x$. $(-2 x+l)^{2}+1=0$.

The condition (3) of Theorem 3 implies $4+\frac{1}{x} \cdot\left(4 l^{2}-\right.$ $4 l)+\frac{1}{x^{2}}\left(-4 l^{3}+l^{2}\right)+\frac{1}{x^{3}}\left(l^{4}+1\right)=0$ and the coefficient $l$ of the line derives from $\psi=4=0$, but asymptote is not obtained.

$c$ has a parabolic branch for $x \rightarrow \infty$ in the direction of the line $y+x=0$.

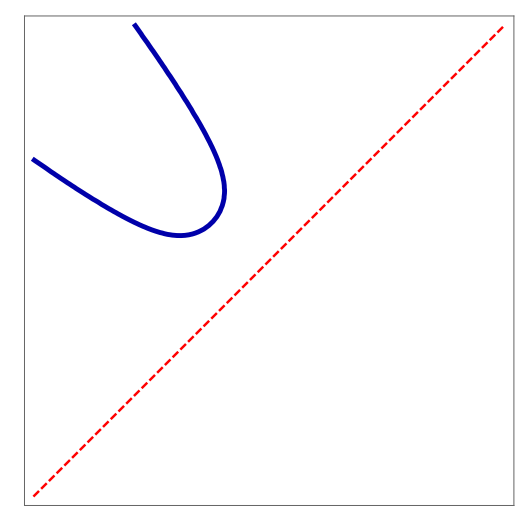

Figure 5: Example 4

We establish the same by Theorem 4. The terms in the equation of the curve are $P_{4}(x, y)=(y-x)^{2}(y+$ $x)^{2}, P_{3}(x, y)=x(y-x)^{2}, P_{2}(x, y)=P_{1}(x, y)=0$. Depending on multiplicity, factors of the leading term are examined to obtain an equation for $t$ as a coefficient in the equation of the line $a x+b y=t$ as a potential asymptote.

- Factor $y-x$ can contribute with power $r=2$ in the point $(1,1)$.

The terms factorise into $P_{4}=(y-x)^{2} \cdot Q_{4}, Q_{4}(x, y)=$ $(y+x)^{2}, P_{3}=(y-x) \cdot Q_{3}, Q_{3}=(y-x)(y+x)$.

The equation for $t$ is $t^{2} \cdot 2^{2}+t \cdot 0 \cdot 2+0=0 \Rightarrow t^{2}=0$. This is a spurious asymptote since $c$ has no a real branch for $x \rightarrow \infty$ in the direction of the line $y-x=$ 0 .

- Factor $y+x$ can contribute with power $r=2$ in the point $(1,-1)$.

The terms factorise into $P_{4}=(y+x)^{2}$. $Q_{4}, Q_{4}(x, y)=(y-x)^{2}$, but $P_{3}$ has no factor $(y+x)$. The power of the factor $y+x$ needs to be reduced to $r=1$ in the same point.

The terms factorise into $P_{4}=(y+x) \cdot Q_{4}, Q_{4}(x, y)=$ $(y+x)(y-x)^{2}$, and the equation for $t$ is $t \cdot 0+4=0$, and asymptote is not obtained.

$c$ has a parabolic branch for $x \rightarrow \infty$ in the direction of the line $y+x=0$.

Following idea from (2) and [20] curve $c$ transforms so that its points at infinity correspond to the origin. In the projective plane, the homogeneous equation of the curve $c$ is $(y-x)^{2}(y+x)^{2}-x(y-x)^{2} z+z^{4}=0$ and homogeneous coordinates of its points at infinity are $[1,1,0]$ and $[1,-1,0]$.

- For the point at infinity $[1,1,0]$, we use the following transformation of coordinates $X \equiv 1, Y \equiv y-x, Z \equiv z$ and the corresponding curve is

$$
\begin{aligned}
& Y^{2}(Y+2)^{2}-Y^{2} Z+Z^{4}=0 \quad \Rightarrow \\
& 4 Y^{2}=-4 Y^{3}+Y^{2} Z-Y^{4}-Z^{4} .
\end{aligned}
$$

But the curve has an isolated point at $(Y, Z)=(0,0)$ and no tangent there.

The curve $c$ does not have a real branch for $x \rightarrow \infty$ in the direction of the line $y-x=0$. Its point at infinity $[1,1,0]$ is an isolated point.

- For the point at infinity $[1,-1,0]$, we use the following transformation of coordinates $X \equiv 1, Y \equiv y+x$, $Z \equiv z$ and the corresponding curve is

$$
\begin{aligned}
& (Y-2)^{2} Y^{2}-(Y-2)^{2} Z+Z^{4}=0 \Rightarrow \\
& 4 Z=4 Y^{2}+4 Y Z-4 Y^{3}-Y^{2} Z+Y^{4}+Z^{4} .
\end{aligned}
$$

The tangent at $(Y, Z)=(0,0)$ is $Z=0$, which corresponds to the line at infinity. There is no asymptote, $c$ has a parabolic branch for $x \rightarrow \infty$ in the direction of the line $y+x=0$.

Example 5 Let $c$ be the curve given by the equation

$$
F(x, y)=x^{4}-2 x^{2} y^{2}+y^{4}+2 x y-2 x^{2}-1=0 .
$$

Following Theorem 3 we find $k_{1}=1, k_{2}=-1$ as the zeros of the leading term $P_{4}(1, t)=(t-1)^{2}(t+1)^{2}$ in the equation of the curve. We substitute for $y=k x+l$ :

- $y=x+l \Rightarrow F(x, x+l)=l^{2}(2 x+l)^{2}+2 x l-1=0$. The condition (3) of Theorem 3 implies $4 l^{2}+$ $\frac{1}{x}\left(4 l^{3}+2 l\right)+\frac{1}{x^{2}}\left(l^{4}-1\right)=0$ and coefficient $l$ of the line derives from $\psi=4 l^{2}=0 \Rightarrow l=0$. $c$ has an asymptote $y-x=0$.

- $y=-x+l \Rightarrow F(x,-x+l)=(-2 x+l)^{2} l^{2}+2 x$. $(-2 x+l)-1=0$.

The condition (3) of Theorem 3 implies $4 l^{2}-4+\frac{1}{x}$. $\left(-4 l^{3}+2 l\right)+\frac{1}{x^{2}}\left(l^{4}-1\right)=0$ and coefficient $l$ of the line derives from $\psi=4 l^{2}-4=0 \Rightarrow l= \pm 1$. $c$ has parallel asymptotes $y=-x-1$ and $y=-x+1$. 


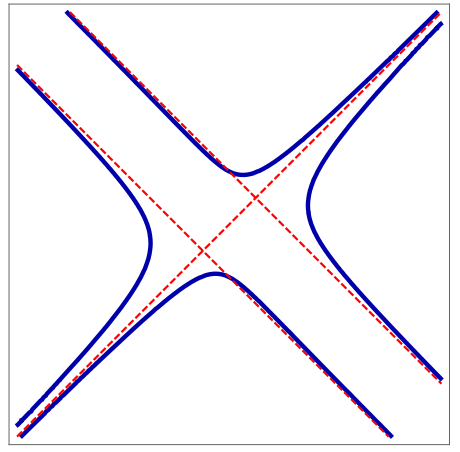

Figure 6: Example 5

We establish the same by Theorem 4 The terms in the equation of the curve are $P_{4}(x, y)=(y-x)^{2}(y+$ $x)^{2}, P_{3}(x, y)=0, P_{2}(x, y)=2 x(y-x), P_{1}(x, y)=0$. Depending on multiplicity, factors of leading term are examined to obtain equation for $t$ as a coefficient in the equation of the line $a x+b y=t$ as potential asymptote.

- Factor $y-x$ can contribute with power $r=2$ in the point $(1,1)$.

The terms factorise into $P_{4}=(y-x)^{2} \cdot Q_{4}$, $Q_{4}(x, y)=(y+x)^{2}, P_{3}=(y-x) \cdot 0$, and the equation for $t$ is $t^{2} \cdot 2^{2}+t \cdot 0+0=0 \Rightarrow t^{2}=0$. $c$ has an asymptote $y-x=0$.

- Factor $y+x$ can contribute with power $r=2$ in the point $(1,-1)$.

The terms factorise into $P_{4}=(y+x)^{2} \cdot Q_{4}$, $Q_{4}(x, y)=(y-x)^{2}, P_{3}=(y+x) \cdot 0$, and the equation for $t$ is $t^{2} \cdot(-2)^{2}+t \cdot 0+(-4)=0 \Rightarrow 4 t^{2}-4=0$. $c$ has parallel asymptotes $y+x-1=0$ and $y+x+$ $1=0$.

Following idea from (2) and [20] curve $c$ transforms so that its points at infinity correspond to the origin. In the projective plane, the homogeneous equation of the curve $c$ is $(y-x)^{2}(y+x)^{2}+2 x(y-x) z^{2}-z^{4}=0$ and homogeneous coordinates of its points at infinity are $[1,1,0]$ and $[1,-1,0]$.

\section{References}

[1] W. W. R. BALL, A Short Account of the History of Mathematics, Dover Publications, 1960.

[2] A. F. Bermant, Course of Mathematical Analysis: Part I, The Macmillan Company, 1963.

[3] W. T. BRANDE, editor, A Dictionary of Science, Literature, and Art: Comprising the History, Description, and Scientific Principles of Every Branch of $\mathrm{Hu}$ -
- For the point at infinity[1,1,0], we use the following transformation of coordinates $X \equiv 1, Y \equiv y-x, Z \equiv z$ and the corresponding curve is

$$
\begin{gathered}
Y^{2}(Y+2)^{2}+2 Y Z^{2}-Z^{4}=0 \quad \Rightarrow \\
4 Y^{2}=-4 Y^{3}-2 Y Z^{2}-Y^{4}+Z^{4} .
\end{gathered}
$$

The curve has tangent $Y=0$ at the cusp $(Y, Z)=$ $(0,0)$, which corresponds to the asymptote $y-x=0$ of the two branches of the curve $c$.

- For the point at infinity $[1,-1,0]$, we use the following transformation of coordinates $X \equiv 1, Y \equiv$ $y+x, Z \equiv z$ and the corresponding curve is

$$
\begin{array}{r}
(Y-2)^{2} Y^{2}+2(Y-2) Z^{2}-Z^{4}=0 \quad \Rightarrow \\
4 Y^{2}-4 Z^{2}=4 Y^{3}-2 Y Z^{2}-Y^{4}+Z^{4} .
\end{array}
$$

The curve has tangents $Y-Z=0$ and $Y+Z=0$ at the node $(Y, Z)=(0,0)$, which corresponds to parallel asymptotes $y-x-1=0$ and $y-x+1=0$ of the curve $c$.

Finally, let us mention that a subtle and so far the most systematic analysis of asymptotes of algebraic curves in real plane, accompanied by an computational algorithm for finding asymptotes by polynomial root isolation was provided by Zeng in [23]. Similarly to the projective geometry approach, he introduced an indeterminate to extend the field $\mathbb{R}$ to a new structure that contains an infinitely large point and keeps the usual ordering and the Euclidean metrics. Based on Sturm sequences and Sturm's theorem, applied to root isolation of the leading polynomial coefficient of the two-variable polynomial defining an algebraic curve in real plane, he developed an algorithm for counting its infinite branches and determining the corresponding asymptotes, if they exist. We omit it here due to its complexity and lack of the space to elaborate its many technical details.

man Knowledge: with the Derivation and Definition of All the Terms in General Use, Harper \& Brothers, 1854.

[4] A. DResden, Introduction to the calculus, Henry Holt and company, 1940.

[5] J. EDWARDS, An elementary treatise on the differential calculus: With applications and numerous examples, Macmillan and Co, 1892. 
[6] R. H. FOWLER, The elementary differential geometry of plane curves, Cambridge, University Press, 1920.

[7] P. Frost, R. J. T. BELL, An elementary treatise on curve tracing, Macmillan, 1918.

[8] P. J. Giblin, What is an Asymptote?, Math. Gaz. 56(398) (1972), 274-284.

[9] G. Glaeser, H. Stachel, B. Odehnal, The Universe of Conics: From the ancient Greeks to 21st century developments, Springer Spektrum, 2016.

[10] W. E. JENNER, Rudiments of algebraic geometry, Oxford University Press, 1963.

[11] A. KatAlenić, A. ČIŽMeŠIJA, Ž. Milin ŠIPUŠ, Asymptote in prospective mathematics teachers' graphing praxeologies, Educação Matemática Pesquisa: Revista do Programa de Estudos PósGraduados em Educação Matemática, 22(4) (2020), 817-826.

[12] A. KatAlenić, A. ČIŽMeŠIJA, Ž. Milin ŠIPUŠ, Prospective mathematics teachers' knowledge of asymptotes and asymptotic behaviour in calculus, International Journal of Science and Mathematics Education, in press.

[13] A. KATAlenić, Ž. Milin ŠIPUŠ, A. ČIŽMEŠIJA, Asymptotes and Asymptotic Behaviour in Graphing Functions and Curves: An Analysis of the Croatian Upper Secondary Education Within the Anthropological Theory of the Didactic, Int J of Sci and Math Educ 18 (2020), 1185-1205.

[14] J. D. KEČKIĆ, A method for obtaining asymptotes of some curves, The teaching of mathematics, III(1) (2000), 53-59.

[15] G. Klambauer, Aspects of Calculus, SpringerVerlag, 1986.

[16] J. Nunemacher, Asymptotes, Cubic Curves, and the Projective Plane, Math. Mag. 3 (1999), 183-192.
[17] T. P. Nunn, Asymptotes, Math. Gaz., 13(182) (1926), 97-103.

[18] C. B. ReAD, What is an Asymptote?, Amer. Math. Monthly 46(8) (1939), 498-499.

[19] J. W. RutTer, Geometry of curves, Chapman \& Hall, 2000.

[20] E. H. SMART, The Nature of an Algebraic Curve at Infinity and the Conic of Closest Contact at Infinity, Math. Gaz. 13(188) (1927), 357-361.

[21] D. E. SMith, History of mathematics, Dover, 1925.

[22] I. TODhunter, A Treatise on the Differential Calculus: With Numerous Examples, Macmillan and Co, 1885.

[23] G. ZENG, Computing the asymptotes for a real plane algebraic curve, Journal of Algebra 316(2) (2007), 680-705.

\author{
Ana Katalenić \\ orcid.org/0000-0001-9857-5758 \\ e-mail: akatalenic@foozos.hr \\ Faculty of Education, University of Osijek \\ Cara Hadrijana 10, HR-31000 Osijek, Croatia

\section{Aleksandra Čižmešija} \\ orcid.org/0000-0002-5017-4143 \\ e-mail: cizmesij@math.hr \\ Faculty of Science, University of Zagreb \\ Bijenička cesta 30, HR-10000 Zagreb, Croatia

\section{Željka Milin Šipuš} \\ orcid.org/0000-0002-0726-3335 \\ e-mail: zeljka.milin-sipus@math.hr \\ Faculty of Science, University of Zagreb \\ Bijenička cesta 30, HR-10000 Zagreb, Croatia
}

